\title{
Lignocellulose biodegradation by Agaricus bisporus during solid substrate fermentation
}

\author{
A. J. Durrant, ${ }^{*}+$ D. A. Wood ${ }^{2}$ and R. B. Cain ${ }^{1}$ \\ ${ }^{1}$ Enviromental Microbiology Laboratory, Department of Agricultural \& Environmental Science, Faculty of Agriculture, \\ The University, Newcastle upon Tyne NE1 7RU, UK \\ ${ }^{2}$ Institute of Horticultural Research, Littlehampton, West Sussex BN17 6LP, UK
}

(Received 28 August 1990; revised 12 December 1990; accepted 20 December 1990)

\begin{abstract}
Agaricus bisporus was cultivated axenically on wheat straw compost. Analysis of this culture medium during growth and fruiting showed that the lignin fraction of straw was degraded preferentially during the vegetative growth phase, whereas cellulose was degraded after the emergence of the fruit bodies. A novel technique was developed, whereby natural or synthetic radiolabelled lignin was mixed intimately with axenic compost and the rate of mineralization to $\mathrm{CO}_{2}$ throughout the life-cycle of $\mathrm{A}$. bisporus was monitored continuously without culture disturbance. Mineralization rates were maximal during the vegetative growth phase and the onset of fruiting brought a decrease of this activity. A mutant strain of $A$. bisporus, which was unable to develop fruiting bodies, was shown to mineralize radiolabelled lignin continuously.
\end{abstract}

\section{Introduction}

The cultivated edible fungus Agaricus bisporus accounts for $70 \%$ of the world annual output of edible mushrooms. The UK alone produces 70000 tonnes (Wood, 1989). A. bisporus is cultivated on a non-sterile but selective compost prepared by microbial treatment of wheat straw. Each year, 300000 tonnes of surplus straw plus stable bedding are converted into mushroom compost (Wood, 1989). This large-scale usage of waste lignocellulosics makes mushroom cultivation one of the few economically viable processes for the bioconversion of lignocelluloses.

The genus Agaricus has long been recognized for its potential to degrade lignocellulosic materials (Waksman \& Nissen, 1931; Gerrits et al., 1967). However, no attempt was made in these early studies to ascertain whether the biodegradation of carbohydrate polymers was occurring in conjunction with lignin biodegradation. Wood \& Leatham (1983) demonstrated that $\boldsymbol{A}$. bisporus could efficiently mineralize a radiolabelled model lignin molecule, namely a dehydrogenative polymer (DHP) of

Present address: Department of Agricultural Biochemistry and Nutrition, The University, Newcastle upon Tyne NE1 7RU, UK.

Abbreviation: DHP, dehydrogenative polymer. coniferyl alcohol ([ring $\left.{ }^{-14} \mathrm{C}\right] \mathrm{DHP}$; Kirk et al., 1975) to ${ }^{14} \mathrm{CO}_{2}$ when cultivated on axenic compost. In these studies no change in the rate of mineralization of lignin was noted throughout the life cycle of $A$. bisporus. These results were obtained by batch sampling from a large commercial-scale crop of mushrooms. Wood \& Goodenough (1977) demonstrated that the onset of fruit body formation brought about large changes in the activity of extracellular enzymes, in particular, polyphenol oxidase, laccase (EC 1.10.3.2) and endocellulase. Laccase has been shown to influence lignin biodegradation in other wood-rotting basidiomycetes, including Phanerochaete chrysosporium (Ander \& Eriksson, 1976) and Coriolus versicolor (Kawai et al., 1988). The precise role of laccase in the degradation of lignin is unknown. The batch sampling method used by Wood \& Leatham (1983) to measure the mineralization of radiolabelled model lignin preparations involved the physical disturbance of the compost and also of the mushroom mycelium, a factor which might have altered the pattern of ligninolysis.

This paper describes the development of a protocol whereby ligninolysis could be monitored continuously throughout the mushroom life cycle and without any physical disturbance to the crop. Because $A$. bisporus is unable to complete its life cycle in liquid culture, this was achieved by mixing axenic mushroom compost with sterile authentic radiolabelled wheat lignocellulose and 
also various model lignin molecules. The mineralization to ${ }^{14} \mathrm{CO}_{2}$ was monitored continuously without physical disturbance to the total solid substrate culture during morphogenesis.

\section{Methods}

Organisms. A. bisporus (Lange) Sing. strain D649 was from the culture collection of the Institute of Horticultural Research, Littlehampton (IHR). This strain was a commercial strain originally obtained from Darlington Mushrooms, Rustington, West Sussex, UK. A. bisporus strain B244 was supplied by IHR. This strain is a mutant which is unable to form fruiting bodies. Both strains were maintained on $2 \%(\mathrm{w} / \mathrm{v})$ malt extract (Boots Pure Drug Co.) solidified with $1.5 \%$ (w/v) Oxoid no. 3 agar. The $\mathrm{pH}$ was adjusted to 5.7 prior to autoclaving at $103 \mathrm{kPa}$ for $15 \mathrm{~min}$. $A$. bisporus was incubated at $25^{\circ} \mathrm{C}$ and subcultured every 6 weeks.

Cultivation of A. bisporus on axenic and non-axenic mushroom compost. A. bisporus was cultivated in $20 \mathrm{~cm} \times 3 \mathrm{~cm}$ tubular growth vessels in an apparatus similar to that designed originally by Long \& Jacobs (1974). Commercial mushroom compost, $30 \mathrm{~g}$ fresh wt (supplied by Turner \& Sons, Stannington, Newcastle upon Tyne, UK) was packed into each tube. For axenic culture, the compost was autoclaved for $30 \mathrm{~m}$ in at 103 $\mathrm{kPa}$ on three successive days. The compost (whether axenic or nonaxenic) was then inoculated with four $/(5 \times 5 \times 5 \mathrm{~mm})$ cubes of malt agar colonized with $A$. bisporus mycelium. Sterile, humidified air delivered from a compressor (Edwards Pumps Ltd.) was then passed through the compost at a flow rate of $30 \mathrm{ml} \mathrm{min}^{-1}$. In accordance with commercial mushroom cultivation procedure (Wood \& Smith, 1987), the cultures were incubated initially at $25^{\circ} \mathrm{C}$. After the mycelium had colonized the compost (spawn run) (15-20 d), a sterile layer of peat/chalk/activated charcoal $(1: 1: 1$, by wt) was added to the surface of the compost (the casing layer) and the incubation temperature reduced to $18{ }^{\circ} \mathrm{C}$ to induce fruit body formation, which was usually evident $20 \mathrm{~d}$ after casing.

Determination of cellulose, hemicellulose, lignin and total nitrogen in mushroom compost. The contents of culture vessels were removed, the casing layer and, where applicable, the fruit bodies were discarded and the compost dried at $105^{\circ} \mathrm{C}$ overnight. To determine the composition of the compost, before, during and after the cultivation of mushrooms, the Van Soest system was used (Van Soest, 1963, 1967) to determine the cellulose and hemicellulose content. Lignin was determined by the method of Johansson et al. (1986) and total nitrogen was determined by the macro-Kjeldahl method.

Preparation of radiolabelled lignins. Samples of $\left[\right.$ ring $\left.^{-1+} \mathrm{C}\right]$, [side-chain$\left.{ }^{14} \mathrm{C}\right]$ and $\left[\right.$ methoxy $\left./{ }^{-14} \mathrm{C}\right] \mathrm{DHPs}$ were provided by $\mathrm{Dr} \mathrm{T}$. K. Kirk (USDA, Forest Service, Forest Products Laboratory, Madison, Wisconsin, USA). These were dissolved in dimethylformamide and filter-sterilized using an autoclaved BAS microfilter fitted with a $0.2 \mu \mathrm{m}$ PTFE filter. Wheat $\left[\right.$ lignin $\left.{ }^{-14} \mathrm{C}\right]$ lignocellulose was provided by Professor E. Odier (INRA, Laboratoire de Microbiologie, Centre de Biotechnologies Agro-industrielles, France). A sterile solution was prepared as detailed for the synthetic lignin.

Addition of radiolabelled lignin into mushroom compost. Sterile solutions of radiolabelled lignin were added to sterile $5 \mathrm{ml}$ aliquots of water, to give $50000 \mathrm{~d}$.p.m. Each aliquot was added to a $30 \mathrm{~g}$ sample of mushroom compost previously packed into a tube. Each tube was sealed, and the radioactive suspension was dispersed throughout the column of compost by forcing a gentle flow of sterile air through for $2 \mathrm{~h}$.
Radiorespirometry. After inoculating each tube with $A$. bisporus (as above) the air outlet was then inserted into a gas-tight air trap and the outlet from this was immersed into a ${ }^{14} \mathrm{CO}_{2}$-trapping cocktail of $7 \mathrm{ml} 2$ ethoxyethanol and $1 \mathrm{ml}$ ethanolamine (Hatakka \& Uusi-Rauva, 1983). Sterile humidified air was forced through the compost $\left(30 \mathrm{ml} \mathrm{min}^{-1}\right)$ and into the trapping fluid. Vials were changed every day. Radioactivity was determined by adding $5 \mathrm{ml}$ of scintillant (Beckman Ready Solv) and assessed by liquid scintillation counting (Beckman LS 3801). All tubing was Butyl-XX, $1.6 \mathrm{~mm}$ wall $\times 5 \mathrm{~mm}$ bore, which was shown to be non-permeable to $\mathrm{CO}_{2}$ even after repeated autoclaving. The tubing and $\mathrm{CO}_{2}$ traps were tested regularly for leakage by examining the recovery of ${ }^{14} \mathrm{CO}_{2}$ from known amounts of acidified sodium $\left[{ }^{14} \mathrm{C}\right]$ bicarbonate. Bacterial air filters (Gelman Sciences) were incorporated into the air inlet lines.

\section{Results}

A. bisporus was cultivated successfully on axenic mushroom compost, within tubular growth chambers. In accordance with standard mushroom production techniques, fruiting body formation was induced by the addition of a layer of peat/chalk/activated charcoal (a casing layer) after $20-25 \mathrm{~d}$ vegetative growth through the compost. Chemical analysis of the composted wheat straw, before, during and after the cultivation of $A$. bisporus (as sole organism) indicated that the cellulose and hemicellulose fractions were degraded preferentially after the addition of the casing layer and the emergence of the fruit bodies (Fig. 1). Total culture nitrogen

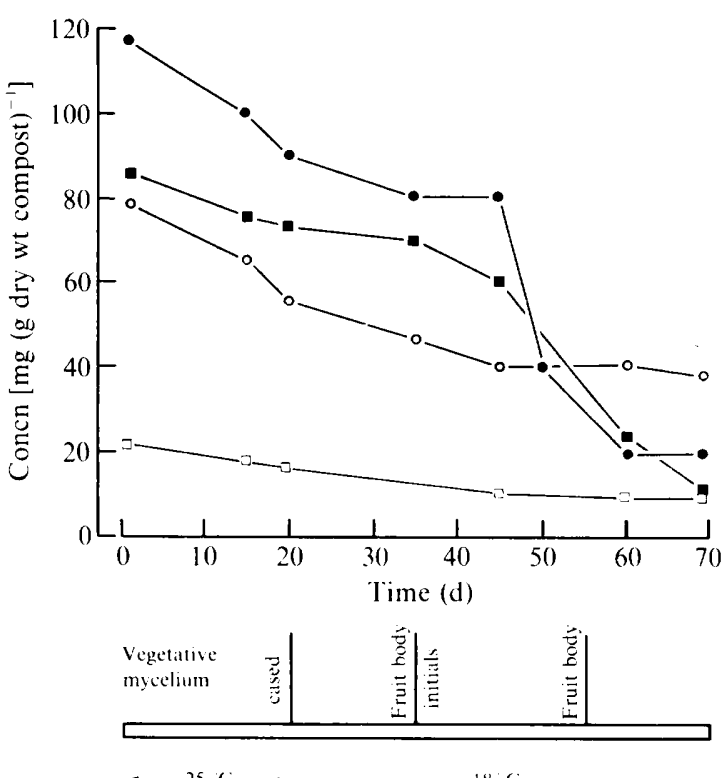

Fig. 1. Biodegradation of lignin $(O)$, cellulose $(\boldsymbol{\square})$ and hemicellulose (C) during the cultivation of $A$. bisporus on axenic compost. Total nitrogen losses $(\square)$ are noted. Chemical analyses were carried out as specified in Methods. Each point on the graph represents the mean value from four samples. Replicates did not vary more than $\pm 6 \%$ about the mean value. 


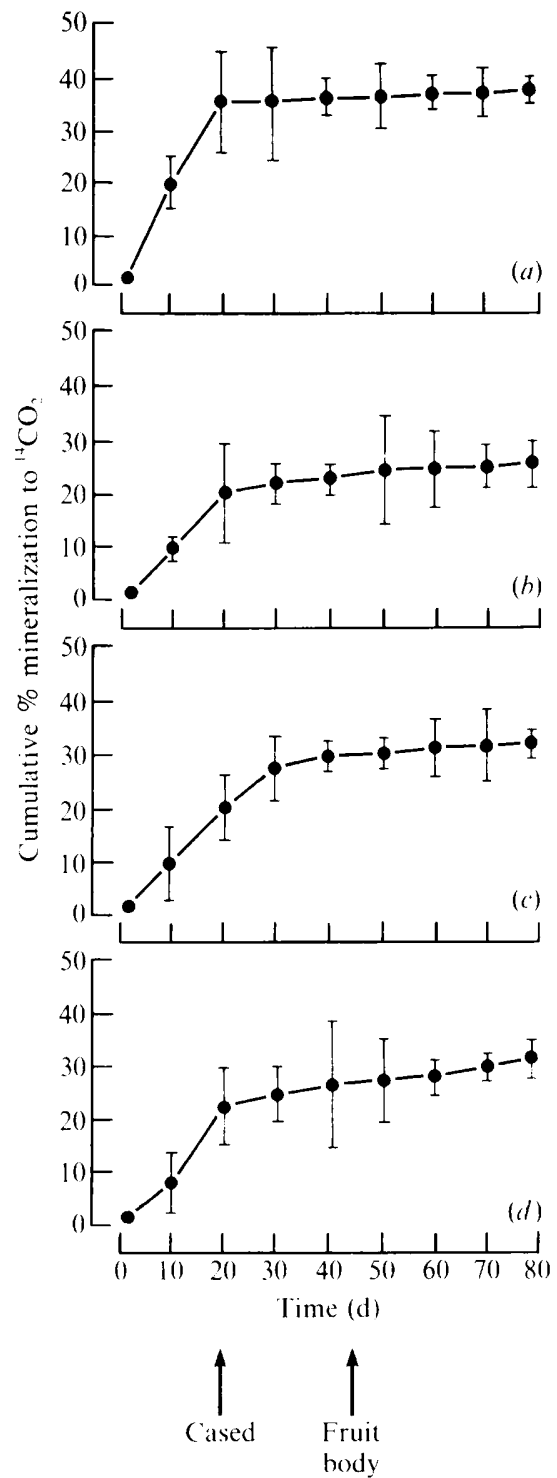

Fig. 2. Mineralization of DHPs and a model lignin dimer by A. hisporus during axenic solid-state fermentation. The radiolabelled compounds and specific activities per vessel were: (a) $\left[\right.$ ring $\left.^{-14} \mathrm{C}\right] \mathrm{DHP}, 48600$ d.p.m.; (b) [side-chain $\left.{ }^{-14} \mathrm{C}\right] \mathrm{DHP}, 42500$ d.p.m.: (c) [ring $\left.A^{-14} \mathrm{C}\right] \beta-\mathrm{O}-4$ dimer, 52000 d.p.m. ; (d) wheat $\left[\right.$ lignin $^{-1+}{ }^{1+}$ C]lignocellulose, 38200 d.p.m. The data represent mean values taken from triplicate tubes.

decreased over the extent of the experiment; the overall rate did not change appreciably after the onset of fruiting. The lignin fraction was degraded preferentially before the addition of the casing layer. To investigate this further, an attempt was made to monitor the mineralization of various radiolabelled lignin preparations when applied to the compost, with the aim of determining which part of the lignin molecule was being degraded preferentially by $A$. bisporus (Fig. 2). In all cases, the preparations were mineralized most extensively to ${ }^{14} \mathrm{CO}_{2}$ during the mycelial colonization. Addition of

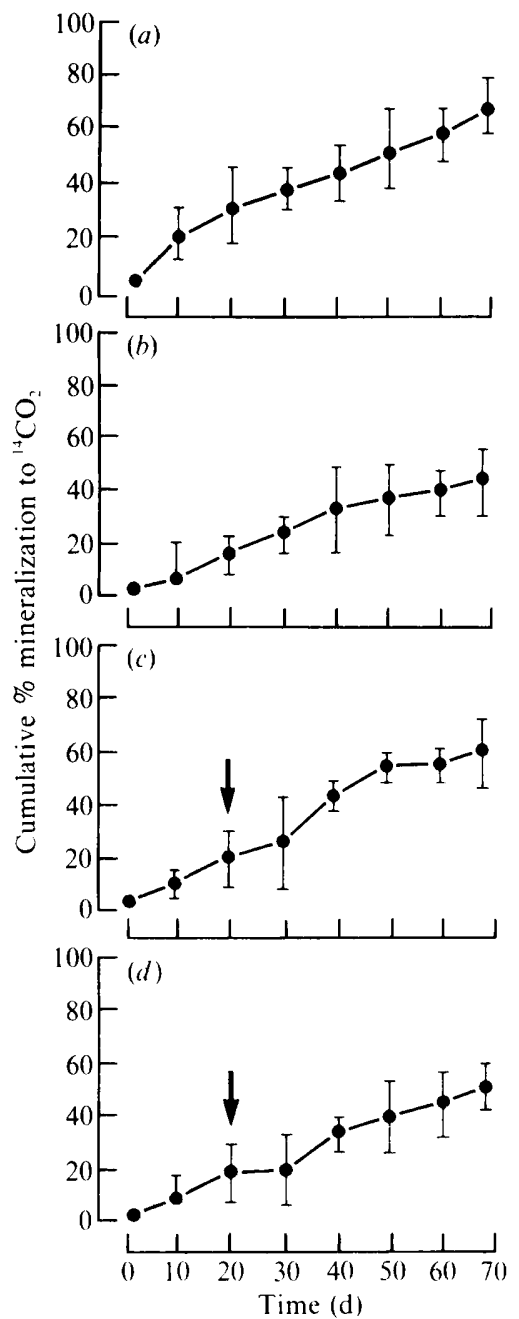

Fig. 3. Mineralization of $\left[\right.$ ring $\left.^{-14} \mathrm{C}\right] \mathrm{DHP}$ (54200 d.p.m. per vessel) (a, $c)$ and wheat $\left[\right.$ lignin $\left.{ }^{-14} \mathrm{C}\right]$ lignocellulose $(38400$ d.p.m. per vessel) $(h, d)$ by a fruit-body-deficient mutant, $A$. bisporus strain B244, during axenic solid-substrate fermentation. $(a, b)$ Uncased; $(c, d)$ cased, denoted by arrows. The data represent mean values taken from triplicate tubes.

the casing layer markedly reduced the ligninolytic activity towards all of the preparations. To determine if this result was simply a physical effect of adding the casing layer or if it was correlated with a genuine physiological switch between the colonization stage and the onset of fruiting, a fruit-body-deficient mutant, $A$. bisporus B244, was used. This mutant was first cultivated similarly on axenic compost and changes in the various lignocellulosic components were determined as described previously for the wild-type strain (results not shown). In all cases, good vegetative mycelial growth was observed, but fruit body initials never appeared. The mycelium of this strain was able to utilize the lignocellulosic fractions at the same rate as the wild-type, but in contrast to the wild-type, no significant difference in the 


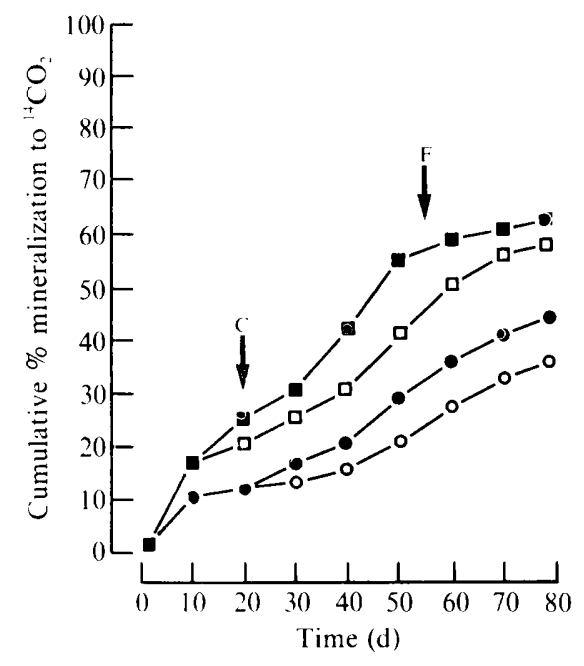

Fig. 4. Mineralization of $\left[\right.$ ring $\left.{ }^{-14} \mathrm{C}\right] \mathrm{DHP}(\square$, cased; $\square$, uncased $)$ and wheat $\left[\right.$ lignin $\left.{ }^{1+} \mathrm{C}\right]$ lignocellulose $(O$, cased; $\boldsymbol{O}$, uncased) by a co-culture of wild-type $A$. bisporus strain D649 and a fruit-body-deficient mutant $A$. bisporus strain B244 during axenic solid-substrate fermentation. The specific activities per vessel were 51600 d.p.m. and 39600 d.p.m. respectively. Arrow $\mathrm{C}$ denotes the addition of a sterile casing layer; fruiting is denoted by arrow $F$. The data represent mean values taken from triplicate tubes. Replicates did not vary more than $11 \%$ about the mean value.

rate of lignin degradation was noted between cased and uncased cultures, suggesting that the process of casing did not itself cause a change in biopolymer utilization. This was confirmed when the mutant was cultivated in the presence of radiolabelled DHP and wheat lignocellulose (Fig. 3). Addition of the casing layer had no significant influence on the mineralization of the preparation to ${ }^{14} \mathrm{CO}_{2}$ by the mutant, in sharp contrast to its effect on the wild-type ligninolytic activity.

To substantiate the evidence for this apparent physiological switch brought about by the onset of fruit body formation upon the ligninolytic activity of $A$. bisporus, the wild-type and the mutant strain were cultivated together on axenic compost in the presence of radiolabelled DHP and wheat lignocellulose (Fig. 4). Fruit body formation occurred but rapid mineralization was still present, showing that the vegetative mycelium of the mutant was still able to effect the mineralization of the radiolabelled DHP and wheat lignin.

\section{Discussion}

Fruit body formation by $A$. bisporus results in a concomitant rise in endocellulase secretion (Wood \& Goodenough, 1977). The increase in enzyme secretion is reflected by the enhanced rates of carbohydrate degradation within the mushroom compost observed in the present study. Total nitrogen losses measured over the total fermentation period were attributed to the release of ammonia and to the exclusion of the mushroom crop during the Kjeldahl digestion. The lignin fraction was degraded primarily during the vegetative growth phase, but hyphal aggregation and the onset of fruiting markedly reduced this degradative capacity. The vegetative growth phase of $A$. bisporus is dominated by the secretion of an extracellular protein, laccase. It has been estimated that as much as $2 \%$ of all protein produced by Agaricus is secreted in the form of laccase (Wood, 1980). Although the precise role of the mushroom laccase in lignin biodegradation is unknown, laccases in general have been implicated, in part, in the degradative capacities of $P$. chrysosporium (Ander \& Eriksson, 1976) and C. versicolor (Kawai, et al., 1988) towards lignin and lignin model compounds. The coincidence of this extracellular enzyme and the onset of lignin biodegradation by $A$. bisporus may be of significance.

Mineralization data confirmed these preliminary observations. All of the radiolabelled preparations were mineralized to ${ }^{14} \mathrm{CO}_{2}$ primarily during the vegetative growth phase. Wheat $\left[\right.$ lignin $\left.^{-14} \mathrm{C}\right]$ lignocellulose was mineralized to a greater extent than would be consistent with the amounts of lignin lost from compost in the biopolymer experiment. It seems likely that the Klason lignin values give only a very conservative estimate of ligninolytic activity, and the product(s) remaining after prolonged acid digestion might be associated with contaminants such as humic substances (Johansson et $a l ., 1986)$. Both the side-chain-labelled and the ringlabelled DHPs were mineralized extensively and the comparable rates displayed by $\left[\right.$ ring $\left.^{-14} \mathrm{C}\right] \mathrm{DHP}$ and the [ring $A^{-14} \mathrm{C}$ ]dimer indicate that the $\beta-O-4$ linkage was at least one of the sites where $A$. bisporus is able to cleave the polymeric lignin. The use of a fruit-body-deficient mutant, $A$. bisporus strain B244, confirmed that lignin biodegradation and mineralization occurred prior to fruit body formation and the use of a co-culture experiment further strengthened these conclusions.

The onset of fruiting brought about by the addition of a casing layer invokes a closely coupled change in both nutritional and developmental physiology in A. bisporus. The biochemical basis for this remains unknown, as does the mechanism for lignin degradation. A. bisporus has shown two previous examples of pronounced regulation of extracellular enzyme activity (Wood \& Goodenough, 1977; Claydon et al., 1988) one of which, for laccase, involved enzyme inactivation (Wood, 1980). It is possible that one or more components of the ligninolytic system of $\boldsymbol{A}$. bisporus may be regulated by inactivation.

That a change in activity was noted, using this particular methodology, strongly implies that the lignin degradative system of $A$. bisporus is sensitive to physical 
disturbance. Comparison of the present data with the results of Wood \& Leatham (1983) implies that physical disturbance of the crop prolongs the expression of ligninolytic activity. This result is seemingly in sharp contrast to the findings of Kirk et al. (1978), who demonstrated that disturbance of a liquid culture of $P$. chrysosporium resulted in mycelial pellet formation and a concomitant decrease in ligninolytic activity. This decrease was attributed to impaired oxygen diffusion into the mycelial pellets. However, a direct comparison with $A$. bisporus is difficult because unlike $P$. chrysosporium, $A$. bisporus is unable to complete its life-cycle in liquid culture. Thus, a true comparison with a physiologically compromised organism is not valid. Cultivation of $\boldsymbol{A}$. bisporus on mushroom compost, where artificial mycelial pellets are unlikely to form, enables it to complete its full life-cycle and the results obtained are probably a better reflection of true ligninolytic activity and its response to physical disturbance.

The biochemical basis of this ligninolytic system coupled with the developmental biology of this economically important higher basidiomycete still remain unclear. Further analysis is in progress to define the process(es) which enable $A$. bisporus to degrade lignin preferentially prior to the emergence of fruiting bodies.

We thank the Science and Engineering Research Council for the award of a CASE studentship to A.J.D.

\section{References}

ANDER, P.\& ERIKSSON, K. E. (1976). The importance of phenol oxidase activity in lignin degradation by the white rot fungus Sporotrichum pulverulentum. Archives of Microbiology 109, 1-8.

Claydon, N., Allan, M. \& Wood, D. A. (1988). Fruit body biomass regulated production of extracellular endocellulase during periodic fruiting by Agaricus bisporus. Transactions of the British Mycological Society 90, 85-90.

Gerrits, J. P. G., Bels-Koning, H. \& Muller, F. M. (1967). Changes in compost constituents during composting, pasteurisation and cropping. Mushroom Science 6, 225-243.

HatakKa \& Uusi-Rauva, U. (1983). Degradation ${ }^{14} \mathrm{C}$-labelled poplar wood lignin by selected white rot fungi. Applied Microbiology and Biotechnology 17, 235-242.

Johansson, M. B., Kogel, I. \& Zeich, W. (1986). Changes in the lignin fraction of spruce and pine needle litter during decomposition as studied by some chemical methods. Soil Biology and Biochemistry 18, 611-619.

Kawal, S., Umezawa, T. \& Higuchi, T. (1988). Degradation mechanisms of phenol $\beta$-1 lignin substructure model compounds by laccase of Coriolus versicolor. Archives of Biochemistry and Biophysics 262, $99-110$.

Kirk, T. K., Connors, W. J., Bleam, R. D., Hackett, W. F. \& ZeIKus, J. G. (1975). Preparation and microbial decomposition of synthetic $\left[{ }^{14} \mathrm{C}\right]$ lignins. Proceedings of the National Academy of Sciences of the United States of America 72, 2515-2519.

Kirk, T. K., Schultz, E., Connors, W. J., Lorenz, L. T. \& Zeikus, J. G. (1978). Influence of culture parameters on lignin metabolism by Phanerochaete chrysosporium. Archives of Microbiology 117, 277-285.

LONG, P. E. \& JACOBS, L. (1974). Aseptic fruiting of the cultivated mushroom Agaricus bisporus. Transactions of the British Mycological Society 67, 99-107.

VAN SOEST, P. J. (1963). The determination of acid detergent fibre. Journal of the Association of Official Agricultural Chemists 46, 829 835 .

VAN SOEST, P. J. (1967). The determination of neutral detergent fibre. Journal of the Association of Official Agricultural Chemists 50, 50-55.

WAKSMAN, S. A. \& NisSEN, W. (1931). Lignin as a nutrient for the cultivated mushroom Agaricus campestris. Science 74, 271-272.

WoOD, D. A. (1980). Inactivation of extracellular laccase during fruiting of Agaricus bisporus. Journal of General Microbiology 117 , 339-345.

WoOD, D. A. (1989). Mushroom biotechnology. International Industrial Biotechnology 9, 5-8.

Wood, D. A. \& Goodenough, P. W. (1977). Fruiting of Agaricus bisporus. Changes in extracellular enzyme activities during growth and fruiting. Archives of Microbiology 114, 161-165.

Wood, D. A. \& Leatham, G. F. (1983). Lignocellulose degradation during the life cycle of Agaricus bisporus. FEMS Microbiology Letters 20, $421-424$.

WoOD, D. A. \& SMITH, J. F. (1987). The cultivation of mushrooms. In Essays in Agricultural and Food Microbiology, pp. 309-343. Edited by J. R. Norris \& G. L. Pettipher. Chichester: John Wiley. 